\title{
Terapia endoscópica combinada en hemorragia diverticular de colon 8
}

\author{
Combined endoscopic therapy for colonic diverticular bleeding
}

Daniel Jaldin Alvarez ${ }^{1}$

\begin{abstract}
La hemorragia diverticular es la causa más frecuente de hemorragia digestiva baja. La hemorragia es abrupta, indolora, abundante. La mayoría de los divertículos que sangran se localizan en el lado derecho, este sangrado se autolimita hasta en un $80 \%$ de los casos. Cuando no se autolimita su manejo puede llegar a ser complejo. El manejo de estos sangrados, pueden variar desde conservador, endoscópico, arteriografía más embolización y el quirúrgico. Entre las alternativas de manejo endoscópico, tenemos la terapia de inyección, la térmica, hemoclips, ligadura con banda. El presente caso es de un paciente con hemorragia diverticular en el que se realizó terapia endoscopica combinada, infiltración de adrenalina, aplicación indirecta de hemoclips y aplicación tópica de ácido tranexámico que es un antifibrinolítico.

Palabras claves: Hemorragia diverticular, hemostasia, adrenalina, hemoclips, acido tranexamico.

\section{Resumen}

Diverticular bleeding is the most common cause of lower Gl bleeding. The bleeding is most often abrupt, painless and abundant. Most of the bleeding diverticula are located on the right side of the colon, this bleeding is self-limited in up to $80 \%$ of cases. When it is not, it could turn into a difficult situation to manage. The management of these bleeds can vary from conservative to an endoscopic, arteriography plus embolization and surgical. Among the endoscopic management alternatives, we have injection therapy, thermal therapy, hemoclips, band ligation. The present case is about a patient with diverticular bleeding who underwent combined endoscopic therapy, adrenaline infiltration, indirect application of hemoclips, and topical application of tranexamic acid, which is an antifibrinolytic.

Keywords: Diverticular bleeding, hemostasis, epinephrine, clipping, tranexamic acid.
\end{abstract}

$\mathrm{L}$

a hemorragia digestiva baja se define como la perdida de sangre a nivel de colon y/o región rectoanal ${ }^{1}$.

La hemorragia diverticular es la causa más frecuente de hemorragia digestiva baja $30-40 \%$, seguido otras causas como sangrado anorectal, patología neoplásica, diversas causas de colitis, angiectasias, ulceras post-polipectomia, etc. Véase (Tabla 1). Se estima que un $15 \%$ de los pacientes con diverticulosis presentaran hemorragia diverticular en algún momento de su vida. Generalmente la hemorragia es abrupta, indolora, y abundante ${ }^{1-3}$.

La etiología del sangrado esta muy relacionado con la fisiopatología de la enfermedad diverticular y la angioestructura del colon, las herniaciones que se producen a nivel del los vasos penetrantes, condiciona que a nivel del fondo diverticular, los vasos rectos que nutren pared del colon estén solo revestidas de una delgada capa de mucosa, lo cual predispone al sangrado(Figura 1$)^{2}$.

La mayoría de los divertículos que sangran se localizan en el lado derecho, este sangrado se autolimita hasta en un $80 \%$ de los casos. Cuando no se autolimita su manejo puede llegar a ser complejo ${ }^{1,2}$.

${ }^{1}$ Médico Gastroenterólogo del Servicio de Endoscopía del I.G.B.J. - CBBA. https://orcid.org/0000-0002-7398-3787

${ }^{*}$ Correspondencia a: Daniel Jaldin Alvarez

Correo electrónico: danieljefd@gmail.com

Recibido el 19 de marzo de 2020. Aceptado el 21 de mayo de 2020.
El manejo de la hemorragia diverticular, puede ir desde un tratamiento conservador a uno endoscópico, arteriografía más embolización e incluso el tratamiento quirúrgico.

Entre las alternativas de manejo endoscópico, tenemos la terapia de inyección, la térmica, hemoclips y la ligadura con banda.

Recientemente se han estado utilizando nuevas técnicas como la ligadura con endoloop y uso de hemospray ${ }^{4-6}$.

El presente caso es de un paciente con hemorragia diverticular al cual se realizó manejo endoscópico del cuadro con una terapia combinada (infiltración de adrenalina,

\begin{tabular}{lc}
\hline \multicolumn{2}{c}{$\begin{array}{c}\text { Causas de hemorragia } \\
\text { digestiva baja }\end{array}$} \\
Lesión & Porcentaje \\
\hline Divertículos & 30 \\
Hemorroides & 14 \\
Colitis isquémica & 12 \\
E. Inflamatoria & \\
Intestinal & 9 \\
Post - Polipectomía & 8 \\
Ca Colon /Pólipos & 6 \\
Ulcera rectal & 6 \\
Angiectasia & 3 \\
Colitis Radica & 3 \\
Otras fuentes & 6 \\
\hline
\end{tabular}

Tabla 1. 


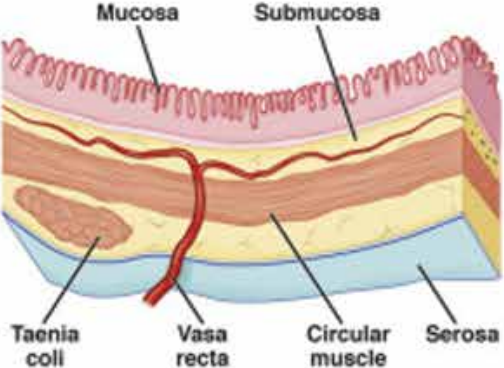

A

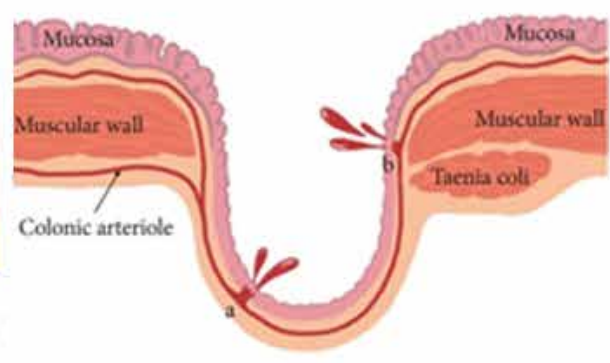

B

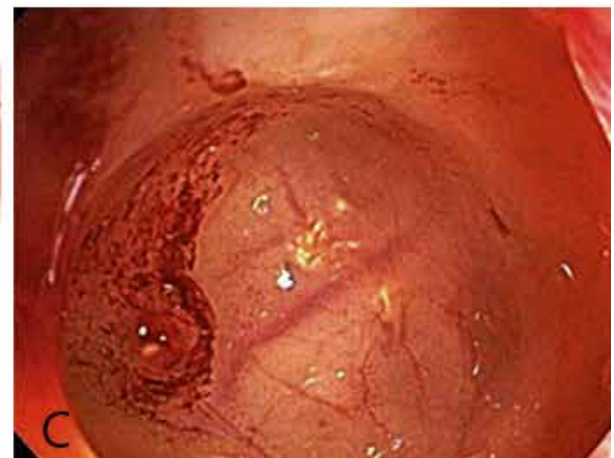

Figura 1. A) Representación de la pared normal de colon. B) Pared de un diverticulo de colon con sangrado. C) Imagen endoscopica de un diverticulo con estigma de sangrado observando a nivel de fondo diverticular

aplicación indirecta de hemoclips y aplicación tópica de ácido tranexámico que es un antifibrinolítico)

\section{Presentación del caso}

Paciente de 75 años de edad ingresa al servicio de emergencias por cuadro de 3 horas de evolución caracterizado por presentar hematoquecia en varias oportunidades, palidez generalizada, moderada alteración del estado hemodinámico.

Paciente tiene antecedentes de hipertensión arterial sistémica, cardiopatía chagásica diagnosticada hace más de 10 años, portador de marcapasos desde hace tres años. Anticoagulación con warfarina, además de consumo de amiodarona y enalapril.

Laboratorios de ingreso hb $11 \mathrm{mg} / \mathrm{dl}$ plaquetas $173000 \mathrm{uL}$ , tiempos de coagulación prolongados INR 2,6 actividad de TP 26 segundos con $27 \%$ de actividad, función renal alterada creatinina $1.8 \mathrm{mg} \mathrm{d} / \mathrm{L}$ BUN 28 se realizó reanimación con cristaloides, vitamina $\mathrm{K}$ EV. preparación para colonoscopia a las 24 hrs. Los laboratorios de control reportaron mejoría de los tiempos de coagulación de 13 segundos actividad del $63 \%$ de actividad INR 1,3 con lo que se realizó colonoscopia evidenciando múltiples divertículos en todo el trayecto del colon con coágulos en su lumen, no se observó sitio de sangrado activo (Figura 2). Con esos resultados se asumio que el sangrado se autolimitó. Después de 24 horas presentó hematoquecia y eliminación de coágulos, descenso de la hb a 8,8 mg dl, alteración del estado hemodinámico, se realizó colonoscopia de urgencia previa preparación con enemas de limpieza y transfusión de concentrado de glóbulos rojos.

Se logró identificar a nivel de colon transverso proximal sangrado rezumante, de uno de los divertículos que no cedió a la infiltración de adrenalina 6cc. A una dilución de 1:10.000 U. Se procedió a marcar el área peridiverticular y contralateral con inyeccion submucosa de azul de metileno y solución fisiológica y posteriormente se ingresa con capuchon. Se procedió a aplicar dos hemoclips recargables (EZ Olympus). no logrando hemostasia satisfactoria se decide irrigar el fondo diverticular ácido tranexámico $500 \mathrm{mg}$ diluido en 20 c.c. de sol fisiológica. a través del canal auxiliar de agua del colonoscopio Olympus CF 180. Cohibiendo el sangrado. Posteriormente se aplicó otros 6 c.c. de adrenalina dil 1:10.000. (Figura 3) (Ver video.)

Posterior al procedimiento paciente tuvo una evolución favorable, hemodinámicamente estable y con deposiciones cafés, por lo que fue egresado a las $72 \mathrm{hrs}$.

\section{Discusión}

La hemorragia diverticular constituye la causa mas frecuente de hemorragia digestiva baja, generalmente se autolimita en hasta un $80 \%$, cuando esto no sucede su manejo puede ser complejo ${ }^{1,2}$. La terapia mas recomendada es la endoscopica, aunque cuándo esta falla , se recomienda

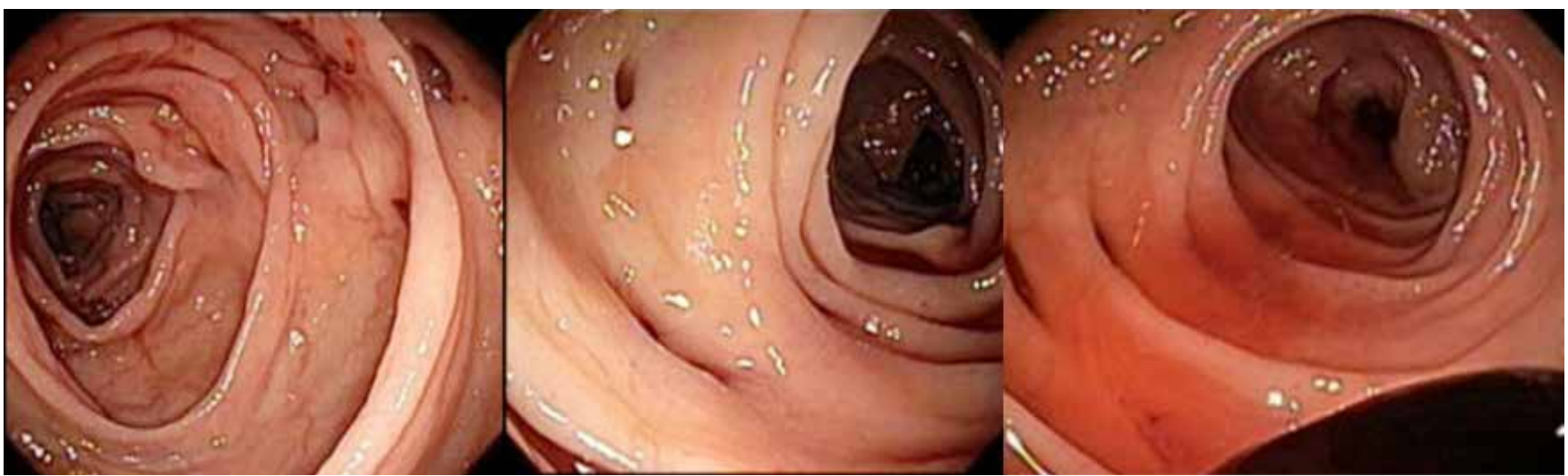

Figura 2. Primera colonoscopia donde se observan múltiples divertículos en el trayecto del colon con coágulos en el lumen, no se observa sangrado activo. 


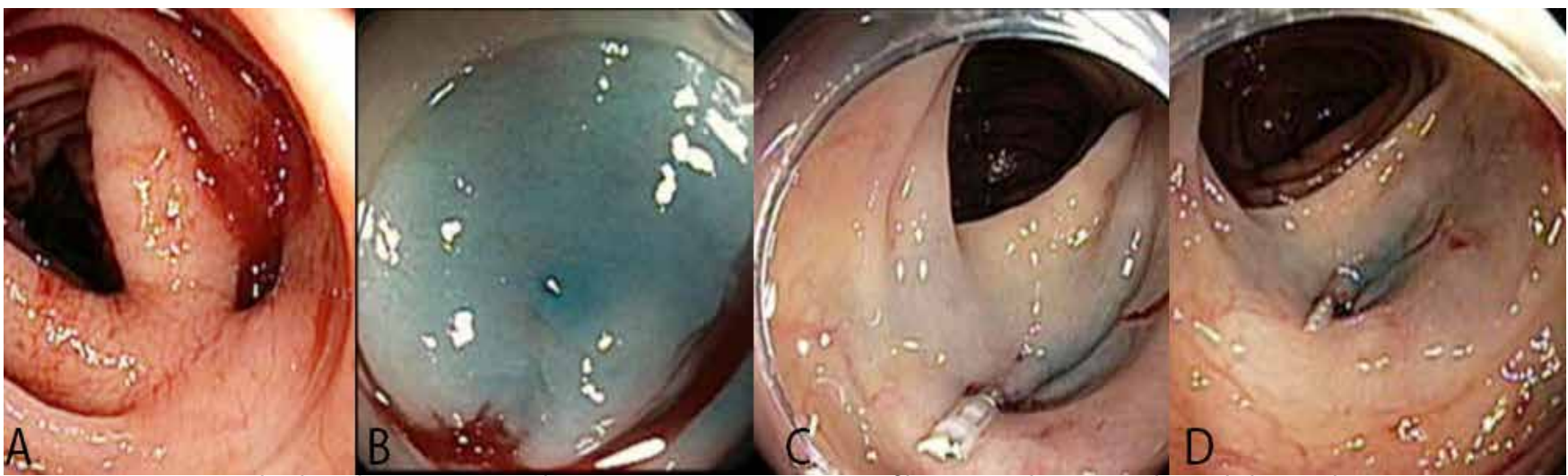

Figura 3. A) Diverticulo de colon con sangrado activo B) Marcacion con infiltración submucosa de azul de metileno C) Ingreso con capuchon y aplicación de endoclip D) Hemostasia posterior a terapia combinada adrenalina, clip, irrigación con ácido tranexámico

la embolizacion arterial y en ultima instancia el tratamiento quirúrgico ${ }^{1,2}$.

Entre las terapias ya mencionadas tenemos la inyección de adrenalina dilución ( 1 en 10000 ). Esta se infiltra a nivel del cuello diverticular. No se recomienda inyectar a nivel de fondo diverticular por el riesgo de perforación debido a la delgadez de la pared a este nivel. Este método tiene un porcentaje alto para lograr un control hemostático temprano, ya que tiene doble efecto tanto de compresión y vasoconstricción, la desventaja es que su efecto disminuye con la reabsorción y cursa con elevadas tasas de resangrado, por lo que no se recomienda la monoterapia con adrenalina ${ }^{4-6}$.

Entre las terapias termicas la mas indicada es la sonda bipolar con resultados similiares a terapias mecánicas como la de los clips y la ligadura, no se recomienda el uso de dispositivos de energía monopolar o argón plasma por el mayor riesgo de lesión transmural y posterior perforación ${ }^{4,5}$.

Entre las terapias mecánicas tenemos al endoclip si bien existen múltiples variedades de clips endoscópicos, dependiendo de la marca y el costo. Los clips mas modernos permiten rotación $360^{\circ}$, reajustar la apertura y cerrado en multiples ocasiones antes de la liberacion del mismo. Existen dos formas de aplicacion de los clip la manera indirecta en la cual se aplican a nivel del cuello del diverticulo a manera de cremallera y la directa en la cual se aplican en el sitio exacto de sangrado. Esta última resulta ser más compleja porque en ocasiones requiere evertir el fondo diverticular, para lo cual son de mucha utilidad los caps ${ }^{4-6}$.

Entre otras terapias mecanicas tenemos la ligadura endoscopica con bandas, se utiliza el mismo dispositivo de ligadura de varices, realizando la misma técnica de aspiración y liberación de la banda a nivel del cuello diverticular, recientemente se ha descrito un procedimiento similar con capuchón y endoloop que tendria como ventaja no tener que cambiar de endoscopio. Este procedimiento cuando está bien realizado tiene alto porcentaje de hemostasia y bajo porcentaje de sangrado. Entre sus desventajas tiene que teóricamente, es que a nivel de colon derecho donde la pared es mas delgada existe mayor probabilidad de ulceraciones y eventual perforación. Además, el tamaño del orificio diverticular puede obstaculizar la técnica cuando este es muy pequeño o muy amplio ${ }^{6,7}$.

Existen tambien dispositivos como el OTSC OVESCO que consiste en un clip tipo trampa de oso adaptado a un capuchon, que permite la eversion del fondo diverticular mediante aspiración, de igual forma que la ligadura endoscopica y posterior liberación del clip. La principal desventaja de este método constituye el elevado coste del dispositivo.

Entre otras tecnicas que se pueden utilizar como terapia adyuvante sellantes de fibrina que son derivados plasmáticos que contienen factores de coagulación en especial factor XIII y trombina. No están disponibles en nuestro medio ${ }^{6,7}$.

Hemospray o endoclot son polvos hemostaticos de formula patentada que al ser aplicados forman un revestimiento que se adhiere al tejido dañado y a su vez interactuan acelerando la cascada de coagulación y por ende la hemostasia, también son de coste alto ${ }^{5,6}$

El ácido tranexamico es un compuesto que logra el bloqueo reversible y competitivo de los receptores de lisina en las moléculas de plasminógeno, impidiendo la unión de éste a la fibrina. Esto reduce la activación del plasminógeno a plasmina con lo que a su vez se inhibe la degradación de la fibrina, que favorece la hemostasia secundaria.

La aplicación intravenosa en horario y oral ha tenido

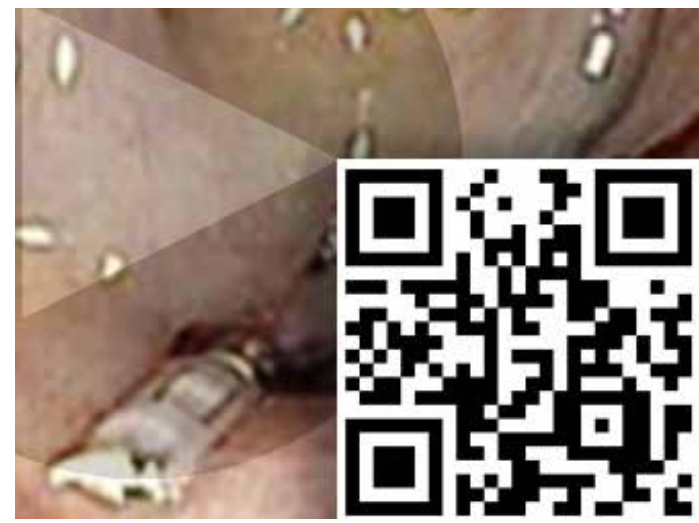

Escanea el código QR para reproducir el Video Enlace del video https://youtu.be/cwtQmBLmCvY 
buenos resultados en hemorragia digestiva alta, logrando retrasar el tiempo de realizacion de la endoscopia alta.

No existen muchos artículos en relacion a la hemorragia digestiva baja, aunque en estos trabajos solo se usó ácido tranexamico intravenoso y via oral.

En trabajos realizados acerca de la aplicación tópica del ácido tranexamico en las áreas de traumatologia y otorrinolaringologia han demostrado efectos favorables en realacion a la hemostasia ${ }^{7-10}$.

Se precisa la realizacion de mas estudios adecuadamente diseñados en relacion al uso topico de acido tranexamico y hemorragia digestiva baja.

En el presente caso se logro la hemostasia combinando metodos de inyeccion, clips y la irrigación topica de acido tranexamico. La cual tuvo un efecto adyuvante beneficioso. Como se ve en el video.

Si bien no existe mucha bibliografia en realacion al uso tópico de ácido tranexámico en hemorragia digestiva baja , podria considerase como una opcion terapeutica adyuvante ya que este farmaco se encuentra disponible en nuestro medio y de bajo coste en relación a otras terapias de rescate.

\section{Referencias bibliográficas}

1. Thomas J. Savides, Dennis M. Jensen, Gastrointestinal Bleeding , Sleisenger and Fordtran's Gastrointestinal and Liver Disease 11th , Philadelphia ,2020, Elsevier.

2. Murphy T, Hunt R, Fried M, et al. Enfermedad Diverticular. World Gastroenterology Organisation Practice Guidelines. 2004

3. Roque Sáenz F . Hemorragia digestiva baja. Gastroenterol. latinoam 2015; Vol 26, Supl No 1 : S $12-S 17$

4. Guardiola J, García-Iglesias P, RodríguezMoranta F, et al. Manejo de la hemorragia digestiva baja aguda: documento de posicionamiento de la Societat Catalana de Digestologia. Gastroenterol Hepatol. 2013;36(8):534-545.
5. Ishii $\mathrm{N}$, Omata $\mathrm{F}$, Nagata $\mathrm{N}$, Kaise $\mathrm{M}$. Effectiveness of endoscopic treatments for colonic diverticular bleeding. Gastrointest Endosc. 2018;87(1):58-66.

6. Nagata N, Ishii N, Manabe N, et al. Guidelines for Colonic Diverticular Bleeding and Colonic Diverticulitis: Japan Gastroenterological Association. Digestion. 2019;99 Suppl 1:1-26.

7. Kobayashi K, Furumoto Y, Akutsu D, Matsuoka M, Nozaka T, Asano T, Fujiki K, Gosho M, Narasaka T, Mizokami Y: Endoscopic Detachable Snare Ligation Improves the Treatment for Colonic Diverticular Hemorrhage. Digestion 2020;101:208-216

8. Montroy J, Hutton B, Moodley P, et al. The efficacy and safety of topical tranexamic acid: A systematic review and meta-analysis [published online ahead of print, 2018 Feb 19]. Transfus Med Rev. 2018;S0887-7963(17)30151-7.

9. Tavakoli N, Mokhtare M, Agah S, et al. Comparison of the efficacy of intravenous tranexamic acid with and without topical administration versus placebo in urgent endoscopy rate for acute gastrointestinal bleeding: A double-blind randomized controlled trial. United European Gastroenterol J. 2018;6(1):4654.

10. Smith SR, Murray D, Pockney PG, Bendinelli C, Draganic BD, Carroll R. Tranexamic Acid for Lower GI Hemorrhage: A Randomized PlaceboControlled Clinical Trial. Dis Colon Rectum. 2018;61(1):99-106. 